\title{
Solution of Second Kind Volterra Integral Equations Using Non-Polynomial Spline Functions
}

\author{
Muna M. Mustafa* \\ Received 20, December, 2012 \\ Accepted 12, June, 2013
}

Sarah H. Harbi*

\begin{abstract}
:
In this paper we use non-polynomial spline functions to develop numerical methods to approximate the solution of $2^{\text {nd }}$ kind Volterra integral equations. Numerical examples are presented to illustrate the applications of these method, and to compare the computed results with other known methods.
\end{abstract}

Key words: Volterra integral equation, non-polynomial spline.

\section{Introduction}

Many problems of mathematical physics can be started in the form of integral equations. These equations also occur as reformulations of other mathematical problems such as partial differential equations and ordinary differential equations. Therefore, the study of integral equations and methods for solving them are very useful in application. In recent years, there has been a growing interest in the Volterra integral equations arising in various fields of physics and engineering[1].

Some valid methods, for solving Volterra equations using various methods have been developed by many researchers, e.g. [1-7]. In this paper, we present for first time a nonpolynomial spline function to introduce numerical method for solving linear Volterra integral equation (VIE) of the second kind.

$u(x)=f(x)+\int_{a}^{x} k(x, t) u(t) d t, \quad a \leq x \leq b \quad .$. (1)

where $k(x, t)$ and $f(x)$ are known functions, but $u(t)$ is an known function. The rest of this paper is organized as follows: In section 2, we will introduce the non-polynomial spline functions. In section 3, we will perform it on Volterra integral equation of the second kind and demonstrate the solving process by discretization. In section 4 , we offers three examples of second kind linear VIE with non-polynomial spline approximation method, and finally section 5 concludes the paper.

\section{Non-polynomial Spline}

\section{Method}

Consider the partition $\Delta=\left\{t_{0}, t_{1}, t_{2}, \ldots, t_{n}\right\}$ of $[a, b] \subset \mathbb{R}$. Let $\mathrm{S}(\Delta)$ denote the set of piecewise polynomials on subinterval $\mathrm{I}_{\mathrm{i}}=\left[\mathrm{t}_{\mathrm{i}}, \mathrm{t}_{\mathrm{i}+1}\right]$ of partition $\Delta$. In this work, we consider non-polynomial spline method for finding approximate solution of VIE of the second kind. Consider the grid point $t_{i}$ on the interval $[a, b]$ as follows:

$$
\begin{aligned}
& a=t_{0}<t_{1}<t_{2}<\ldots<t_{n}=b \\
& t_{i}=t_{0}+i h, i=0,1, \ldots, n \\
& h=\frac{b-a}{n}
\end{aligned}
$$

where $\mathrm{n}$ is a positive integer. Let $u(t)$ be the exact solution of (1) and $S_{i}(t)$ be an approximate to $u_{i}=u\left(t_{i}\right)$ obtained by the segment $P_{i}(t)$. Each nonpolynomial spline segment $P_{i}(t)$ has the form[8]:

* College Science for women, Baghdad University, Al-Jadriyah, Baghdad,Iraq. 
(5)

where $a_{i}, b_{i}, c_{i}$, and $d_{i}$ are constant and $k$ is the frequency of the trigonometric functions which will be used to raise the accuracy of the method. We consider the following relations:

$P_{i}\left(t_{i}\right)=a_{i}+d_{i}=u\left(t_{i}\right) \approx S_{i}\left(t_{i}\right)$

$\mathrm{P}^{s}{ }_{\mathrm{i}}\left(\mathrm{t}_{\mathrm{i}}\right)=\mathrm{kb}_{\mathrm{i}}+\mathrm{c}_{\mathrm{i}}=\mathrm{u}^{\prime}\left(\mathrm{t}_{\mathrm{i}}\right) \approx S_{i}^{\prime}\left(t_{i}\right)$

$\mathrm{P}^{s s}\left(\mathrm{t}_{\mathrm{i}}\right)=-\mathrm{k}^{2} \mathrm{a}_{\mathrm{i}}=\mathrm{u}^{s s}\left(\mathrm{t}_{\mathrm{i}}\right) \approx S_{i}^{\prime \prime}\left(t_{i}\right)$

$\mathrm{P}^{\mathrm{sss}}{ }_{\mathrm{i}}\left(\mathrm{t}_{\mathrm{i}}\right)=-\mathrm{k}^{3} \mathrm{~b}_{\mathrm{i}}=\mathrm{u}^{\mathrm{a} w}\left(\mathrm{t}_{\mathrm{i}}\right) \approx S_{i}^{\mathrm{m}}\left(t_{i}\right)$

We can obtain the values of $\mathrm{a}_{\mathrm{i}}, \mathrm{b}_{\mathrm{i}}, \mathrm{c}_{\mathrm{i}}$, and $\mathrm{d}_{\mathrm{i}}$ as follows:

$a_{i}=-\frac{1}{k^{2}} u^{m}\left(t_{i}\right) \approx-\frac{1}{k^{2}} S_{i}^{w}\left(t_{i}\right) \ldots$

$\mathrm{b}_{\mathrm{i}}=-\frac{1}{\mathrm{k}^{\mathrm{s}}} \mathrm{u}^{s s s}\left(\mathrm{t}_{\mathrm{i}}\right) \approx-\frac{1}{\mathrm{k}^{\mathrm{s}}} S_{i \mathrm{i}}^{\mathrm{w}}\left(\mathrm{t}_{\mathrm{i}}\right)$ $\mathrm{c}_{\mathrm{i}}=\mathrm{u}^{\prime}\left(\mathrm{t}_{\mathrm{i}}\right)+\frac{1}{\mathrm{k}^{2}} \mathrm{u}^{s s}\left(\mathrm{t}_{\mathrm{i}}\right) \approx S_{i}^{\prime}\left(\mathrm{t}_{\mathrm{i}}\right)+\frac{1}{\mathrm{k}^{2}} S_{i}^{\prime \prime \prime}\left(\mathrm{t}_{\mathrm{i}}\right)$

... (8)

$\mathrm{d}_{\mathrm{i}}=\mathrm{u}\left(\mathrm{t}_{\mathrm{i}}\right)+\frac{1}{\mathrm{k}} \mathrm{u}^{* s}\left(\mathrm{t}_{\mathrm{i}}\right) \approx \mathrm{S}_{\mathrm{i}}\left(\mathrm{t}_{\mathrm{i}}\right)+\frac{1}{\mathrm{k}} S_{i}^{\prime \prime}\left(\mathrm{t}_{\mathrm{i}}\right)$

for $i=0,1, \ldots, n$.

\section{The Solving Method}

Consider the linear VIE of the second kind (1), in order to solve (1), we differentiate (1) three times with respect to $\mathrm{x}$ and then put $\mathrm{x}=\mathrm{a}$, to get:

$\mathrm{u}_{0}=\mathrm{u}(\mathrm{a})=\mathrm{f}(\mathrm{a})$

... (10)

$\mathrm{u}_{0}^{\prime}=\mathrm{u}^{\prime}(\mathrm{a})=\mathrm{f}^{\prime}(\mathrm{a})+\mathrm{k}(\mathrm{a}, \mathrm{a}) \mathrm{u}(\mathrm{a})$

$\mathrm{u}_{0}^{s s}=\mathrm{u}^{s s}(\mathrm{a})=\mathrm{f}^{s s}(\mathrm{a})+\left(\left.\frac{\partial \mathrm{k}(\mathrm{x}, \mathrm{t})}{\partial \mathrm{x}}\right|_{\mathrm{t}=\mathrm{x}}\right)_{\mathrm{x}=\mathrm{a}} \mathrm{u}(\mathrm{a})+\left.\frac{\mathrm{dk}(\mathrm{x}, \mathrm{x})}{\mathrm{dx}}\right|_{\mathrm{x}=\mathrm{a}} \mathrm{u}(\mathrm{a})+\mathrm{k}(\mathrm{a}, \mathrm{a}) \mathrm{u}^{\prime}(\mathrm{a}) \cdots($

$\mathrm{u}_{0}^{\mathrm{sss}}=\mathrm{u}^{\mathrm{sss}}(\mathrm{a})=$

$\mathrm{f}^{s s}(\mathrm{a})+\left(\left.\frac{\partial^{\mathrm{z}} \mathrm{k}(\mathrm{x}, \mathrm{t})}{\partial \mathrm{x}^{2}}\right|_{\mathrm{t}=\mathrm{x}}\right) \mathrm{x}=\mathrm{a}_{\mathrm{s}} \mathrm{u}(\mathrm{a})+\left(\frac{\mathrm{d}}{\mathrm{dx}}\left(\left.\frac{\partial \mathrm{k}(\mathrm{x}, \mathrm{t})}{\partial \mathrm{x}}\right|_{\mathrm{t}=\mathrm{x}}\right)\right)_{\mathrm{x}=\mathrm{a}} \mathrm{u}(\mathrm{a})+\left(\left.\frac{\partial \mathrm{k}(\mathrm{x}, \mathrm{t})}{\partial \mathrm{x}}\right|_{\mathrm{t}=\mathrm{x}}\right) \mathrm{x}=\mathrm{a} \mathrm{u}^{\prime}(\mathrm{a})+$

$\left.\frac{d^{2} k(x, x)}{d x^{2}}\right|_{x=a} u(a)+\left.2 \frac{d k(x, x)}{d x}\right|_{x=a^{\prime}} u^{\prime}(a)+k(a, a) u^{w s}(a)$

Therefore, we approximate the solution of (1) using (5) following algorithm (VIENPS):

\section{Algorithm (VIENPS)}

To find the approximate solution of (1), first we select positive integer $\mathrm{n}$, and perform the following steps:

Step 1: Set $\mathrm{h}=(\mathrm{b}-\mathrm{a}) / \mathrm{n}$; $\mathrm{t}_{\mathrm{i}}=\mathrm{t}_{0}+\mathrm{ih}, \mathrm{i}=0,1, \ldots, \mathrm{n}, \mathrm{t}_{0}=\mathrm{a}, \mathrm{t}_{\mathrm{n}}=\mathrm{b}$ and $\mathrm{u}_{0}=\mathrm{f}(\mathrm{a})$

Step 2: Evaluate $a_{0}, b_{0}, c_{0}$ and $d_{0}$ by substituting 10-13 in equations 6-9.

Step 3: Calculate $P_{0}(t)$ using step 2 and equation 5 for $i=0$.

Step 4: Approximate $\mathrm{u}_{1} \approx \mathrm{P}_{0}\left(\mathrm{t}_{1}\right)$.

Step 5: For $i=1$ to $n-1$ do the following steps:

Step 6: Evaluate $a_{i}, b_{i}, c_{i}$ and $d_{i}$ using equations 6-9 and replacing $\mathrm{u}\left(\mathrm{t}_{\mathrm{i}}\right)$, $\mathrm{u}^{\prime}\left(\mathrm{t}_{\mathrm{i}}\right), \mathrm{u}^{s s}\left(\mathrm{t}_{\mathrm{i}}\right)$ and $\mathrm{u}^{s w}\left(\mathrm{t}_{\mathrm{i}}\right)$ by $\mathrm{P}_{\mathrm{i}}\left(\mathrm{t}_{\mathrm{i}}\right)$, $\mathrm{P}_{\mathrm{i}}^{*}\left(\mathrm{t}_{\mathrm{i}}\right), \mathrm{P}_{\mathrm{i}}^{s s}\left(\mathrm{t}_{\mathrm{i}}\right)$ and $\mathrm{P}_{\mathrm{i}}^{\text {sss }}\left(\mathrm{t}_{\mathrm{i}}\right)$.

Step 7: Calculate $P_{i}(t)$ using step 6 and equation 5 .

Step 8: Approximate $u_{i+1}=P_{i}\left(t_{i+1}\right)$.

\section{Numerical Examples}

4.1 Example 1: Consider VIE of second kind [1]:

$\emptyset(x)=x+\int_{0}^{x}(t-x) \emptyset(t) d t, 0 \leq x \leq 1$

the exact solution is $\emptyset(x)=\sin x$. Results have been shown in Table 1, where $P_{i}(x)$ denote the approximate solution by the proposed method and $\operatorname{err}=\left|\emptyset(x)-\mathrm{P}_{\mathrm{i}}(\mathrm{x})\right|$. 
Table 1: Computed Absolute Error of Example (1) and The Result Obtain in [1]

\begin{tabular}{|l|l|l|l|l|}
\hline $\mathbf{x}$ & Exact Solution $\emptyset(\boldsymbol{x})$ & $\mathbf{P}_{\mathbf{i}}(\mathbf{x})$ & error & error obtain in [1] \\
\hline $\mathbf{0 . 0}$ & 0 & 0 & 0 & 0 \\
\hline $\mathbf{0 . 1}$ & 0.099833416646828 & 0.099833416646828 & 0 & $2.0508063 \mathrm{e}-012$ \\
\hline $\mathbf{0 . 2}$ & 0.198669330795061 & 0.198669330795061 & 0 & $8.3558996 \mathrm{e}-013$ \\
\hline $\mathbf{0 . 3}$ & 0.295520206661340 & 0.295520206661340 & $5.551115123 \mathrm{e}-017$ & $2.0756558 \mathrm{e}-013$ \\
\hline $\mathbf{0 . 4}$ & 0.389418342308651 & 0.389418342308650 & $1.110223024 \mathrm{e}-016$ & $2.4960310 \mathrm{e}-013$ \\
\hline $\mathbf{0 . 5}$ & 0.479425538604203 & 0.479425538604203 & $1.110223024 \mathrm{e}-016$ & $3.6565473 \mathrm{e}-013$ \\
\hline $\mathbf{0 . 6}$ & 0.564642473395035 & 0.564642473395035 & $1.110223024 \mathrm{e}-016$ & $1.5317015 \mathrm{e}-013$ \\
\hline $\mathbf{0 . 7}$ & 0.644217687237691 & 0.644217687237691 & $1.110223024 \mathrm{e}-016$ & $1.190846 \mathrm{e}-013$ \\
\hline $\mathbf{0 . 8}$ & 0.717356090899523 & 0.717356090899523 & $2.220446049 \mathrm{e}-016$ & $2.5211375 \mathrm{e}-013$ \\
\hline $\mathbf{0 . 9}$ & 0.783326909627483 & 0.783326909627483 & $3.330669073 \mathrm{e}-016$ & $2.8431391 \mathrm{e}-013$ \\
\hline $\mathbf{1 . 0}$ & 0.841470984807897 & 0.841470984807896 & $4.440892098 \mathrm{e}-016$ & $8.8095244 \mathrm{e}-013$ \\
\hline
\end{tabular}

4.2 Example 2: Consider VIE of second kind $[2,4]$ :

$y(x)=1+\int_{0}^{x}(t-x) y(t) d t, 0 \leq x \leq 1$ the exact solution is $y(x)=\cos x$. Results have been shown in Table 2, where $\mathrm{P}_{\mathrm{i}}(\mathrm{x})$ denote the approximate solution by the proposed method.

Table 2: Computed Absolute Error of Example (2) and The Result Obtain in [2] and [4]

\begin{tabular}{|l|l|l|l|l|l|}
\hline $\mathbf{x}$ & Exact Solution $\boldsymbol{y}(\boldsymbol{x})$ & $\mathbf{P}_{\mathbf{i}}(\mathbf{x})$ & error & error obtain in [4] & error obtain in [2] \\
\hline $\mathbf{0 . 0}$ & 1.000000000000000 & 1.000000000000000 & 0 & - & 0 \\
\hline $\mathbf{0 . 1}$ & 0.995004165278026 & 0.995004165278026 & 0 & $9.97 \mathrm{e}-08$ & - \\
\hline $\mathbf{0 . 2}$ & 0.980066577841242 & 0.980066577841242 & $1.11022302462 \mathrm{e}-016$ & $4.16 \mathrm{e}-07$ & 0 \\
\hline $\mathbf{0 . 3}$ & 0.955336489125606 & 0.955336489125606 & $1.11022302462 \mathrm{e}-016$ & $8.77 \mathrm{e}-07$ & - \\
\hline $\mathbf{0 . 4}$ & 0.921060994002885 & 0.921060994002885 & $2.22044604925 \mathrm{e}-016$ & $1.58 \mathrm{e}-06$ & 0 \\
\hline $\mathbf{0 . 5}$ & 0.877582561890373 & 0.877582561890373 & $2.22044604925 \mathrm{e}-016$ & $6.76 \mathrm{e}-07$ & - \\
\hline $\mathbf{0 . 6}$ & 0.825335614909678 & 0.825335614909678 & $2.22044604925 \mathrm{e}-016$ & $3.35 \mathrm{e}-06$ & $8.993 \mathrm{e}-015$ \\
\hline $\mathbf{0 . 7}$ & 0.764842187284489 & 0.764842187284488 & $3.33066907387 \mathrm{e}-016$ & $4.48 \mathrm{e}-06$ & - \\
\hline $\mathbf{0 . 8}$ & 0.696706709347165 & 0.696706709347165 & $3.33066907387 \mathrm{e}-016$ & $5.52 \mathrm{e}-06$ & $5.031 \mathrm{e}-013$ \\
\hline $\mathbf{0 . 9}$ & 0.621609968270664 & 0.621609968270664 & $3.33066907387 \mathrm{e}-016$ & $6.80 \mathrm{e}-06$ & - \\
\hline $\mathbf{1 . 0}$ & 0.540302305868140 & 0.540302305868139 & $4.44089209850 \mathrm{e}-016$ & $2.61 \mathrm{e}-04$ & $1.142 \mathrm{e}-011$ \\
\hline
\end{tabular}

4.3 Example 3: Consider VIE of second kind [1]:

$u(x)=2 x+5-3 e^{x}+\int_{0}^{x} e^{x-t} u(t) d t, 0 \leq x \leq 1$

the exact solution is $u(x)=x+1$.

solution by the proposed method and Results have been shown in Table 3, $\operatorname{err}=\left|\emptyset(x)-\mathrm{P}_{\mathrm{i}}(\mathrm{x})\right|$. where $P_{i}(x)$ denote the approximate

Table 3: Computed Absolute Error of Example (3)

\begin{tabular}{|l|l|l|l|}
\hline $\mathbf{x}$ & Exact Solution $\boldsymbol{u}(\boldsymbol{x})$ & $\mathbf{P}_{\mathbf{i}}(\mathbf{x})$ & error \\
\hline $\mathbf{0 . 0}$ & 2.000000000000000 & 2.000000000000000 & 0 \\
\hline $\mathbf{0 . 1}$ & 2.100000000000000 & 2.100000000000000 & 0 \\
\hline $\mathbf{0 . 2}$ & 2.200000000000000 & 2.200000000000000 & 0 \\
\hline $\mathbf{0 . 3}$ & 2.300000000000000 & 2.300000000000000 & $4.440892098500 \mathrm{e}-016$ \\
\hline $\mathbf{0 . 4}$ & 2.400000000000000 & 2.400000000000000 & $4.440892098500 \mathrm{e}-016$ \\
\hline $\mathbf{0 . 5}$ & 2.500000000000000 & 2.500000000000000 & $4.440892098500 \mathrm{e}-016$ \\
\hline $\mathbf{0 . 6}$ & 2.600000000000000 & 2.600000000000001 & $4.440892098500 \mathrm{e}-016$ \\
\hline $\mathbf{0 . 7}$ & 2.700000000000000 & 2.700000000000001 & $4.440892098500 \mathrm{e}-016$ \\
\hline $\mathbf{0 . 8}$ & 2.800000000000000 & 2.800000000000001 & $8.881784197001 \mathrm{e}-016$ \\
\hline $\mathbf{0 . 9}$ & 2.900000000000000 & 2.900000000000001 & $8.881784197001 \mathrm{e}-016$ \\
\hline $\mathbf{1 . 0}$ & 3.000000000000000 & 3.000000000000001 & $8.881784197001 \mathrm{e}-016$ \\
\hline
\end{tabular}

\section{Conclusion}

In this paper, non-polynomial spline function method for solving Volterra integral equations of the second kind is presented successfully. This new idea based on the use of the VIE and its derivatives. So it is necessary to mention that this approach 
can be used when $\mathrm{f}(\mathrm{x})$ and $\mathrm{k}(\mathrm{x}, \mathrm{t})$ are analytic. The proposed scheme is simple and computationally attractive and their accuracy are high and we can execute this method in a computer simply. The numerical examples support this claim.

\section{References}

1. M. M. Rahman, M. A. Hakim, M. Kamrul Hasan, M. K. Alam and L. Nowsher, 2012, Numerical Solution of Volterra Integral Equations of Second Kind with the Help of Chebyshev Polynomials, Annals of Pure and Applied Mathematics, 1(2): 158-167.

2. A. Tahmasbi, 2008, A New Approach to the Numerical Solution of Linear Volterra Integral Equations of the Second Kind, Int. J. Contemp. Math. Sciences, 3(32): 1607-1610.

3. J. Rashidinia and M. Zarebnia, 2008, New Approach for Numerical Solution of Volterra Integral Equations of the second Kind, IUST International Journal of Engineering Science, 19(52): 59-65.

4. M. I. Berenguer, D. Gámez, A. I. Garralda-Guillen, M. Ruiz Galán, and
M. C. Serrano Pérez, 2009, Analytical techniques for a numerical solution of the linear Volterra integral equation of the second kind, Hindawi Publishing Corporation, doi:10.1155/2009/149367.

5. K. Maleknejad, E. Hashemizadeh and R. Ezzati, 2011, A new approach to the numerical solution of Volterra integral equations by using Bernstein's approximation, Commun Nonlinear Sci Numer Simulat, 16: 647-655.

6. J. Biazar and M. Eslami, 2011, Homotopy perturbation and Taylor series for Volterra integral equations of the second kind, Middle-East Journal of Scientific Research, 7(4): 604-609.

7. F. Mirzaee, 2012, A computational Method for Solving Linear Volterra Integral Equations, Applied Mathematical Sciences, 6(17): 807814.

8. M. Zarebnia, M. Hoshyar and M. Sedaghti, 2011, Non-polynomial spline method for the solution of problems in calculus of variations, World Academy of science, Engineering and Technology, 51: 986-991.

\section{حل معادلات فولتيرا التكاملية من النوع الثاني باستخدام دوال الثلمة الغير متعددة الحدود النو
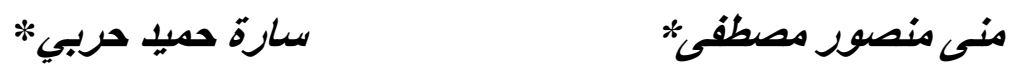 \\ | - ملية العلوم للبنات، جامعة بغداد، الجادرية، بغداد، العراق.

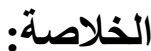 \\ في هذا البحث تم استخدام دو ال الثلمة الغير متعددة الحدود لايجاد حل عددي تقريبي لمعادلات فولتبرا التكاملية من النوع الثاني. تم اعطاء امثلة عددية لتوضيح تطبيق الطريقة، كما تم مقارنة النتائج مع طرق الخرى لثرى معروفة.}

